\title{
A Preliminary Investigation of Adult Defence Style and Physiological Reactivity to Infant Distress Signals
}

\author{
Emma L. Gould, Tammy Lane, Andrew J. Lewis \\ Deakin University, Geelong, Australia
}

\begin{abstract}
Species whose offspring require extended care-giving ought to be predisposed to being biologically responsive to their infant's signalling. This paper examined the interplay between biological and psychological aspects of adult response to an infant's distress. HR (heart rate) and GSR (galvanic skin response) were recorded continuously, while 50 adults listened to white noise and an infant cry audio recording. Participants completed the defence style questionnaire and the state trait anxiety inventory. HR acceleration occurred in response to the control sound, while HR decelerated in response to the infant cry. GSR responsiveness was positively correlated with immature and neurotic defence styles. When controlling for other variables, immature defence was a unique and independent predictor of GSR change in response to infant distress. Defence demonstrated a stronger relationship than self-reported anxiety, than that with physiological responsiveness. Employing defence mechanisms appears to reduce an individual's perceived anxiety, though it has little effect on physiological arousal levels.
\end{abstract}

Keywords: infant distress signals, physiological responsiveness, defence mechanisms, galvanic skin response, HR (heart rate)

\section{Introduction}

Variation exists in how adults respond to infant signalling behaviour (McGregor \& Peake, 2000). In infants, signalling behaviour is exhibited through a range of behaviors, such as crying, smiling, following and use of gaze. Infant crying is a marker of distress or danger and ought to be associated with rapid adult response. Variation in adult responsiveness to infant distress has been shown to have implications for the quality of caregiving, in turn impacting on the development of the child's attachment behavioral system and socio-emotional development. Defence mechanisms are automatic psychological processes that protect against anxiety and awareness of internal and external dangers (Bond, 2004). This study examines the extent to which defence mechanisms influence adult responsiveness to infant signaling. Since defences are thought to be a means of cognitively regulating either perceived anxiety or physiological arousal reflecting anxiety states, this study examines the interaction of both physiological and psychological variables in response to infant distress cries.

Defence mechanisms have been found to cluster into styles that can be ranked on a developmental continuum from mature or adaptive defences, to maladaptive defences, with neurotic defence falling in between (Bond, 2004). Maladaptive defences have been found to be correlated with more severe psychopathology than

Emma L. Gould, School of Psychology, Deakin University.

Tammy Lane, School of Psychology, Deakin University.

Andrew J. Lewis, Ph.D., associate professor, School of Psychology, Deakin University. 
adaptive defences (Nishimura, 1998). Mature defences have been defined as appropriate, realistic ways of dealing with painful feelings brought on by emotional conflict or stressors, whether they be internal or external (Cohen, 2003). These include responses to life stressors or anxiety, such as the use of humor, seeking social affiliation or support, or directing one's attention to creative modes for the expression of emotion, known as sublimation. In contrast, immature defences are more commonly observed earlier in development and are associated with forms of psychopathology or interpersonal conflict (Lewis \& White, 2009). These include the denial of the stressor, projection of the cause of the distress onto another person or alternating between idealization and denigration within interpersonal relations, known as splitting. Such defences frequently involve a blurring between the self and the outer world (Huprich, 2008). Finally, neurotic defences produce some forms of ineffective coping and may cause a significant level of psychological distress. Neurotic defences include repression, dissociation or reaction formation, where the individual acts or behaves in the opposite manner to what they want or feel (Barry \& Farmer, 2001). More specifically, mature defence styles are thought to represent the successful use of defence mechanisms, and effectively relieve anxiety and distress. In contrast, neurotic defence styles are often accompanied by a short-term success in regulating anxiety and distress. Immature defences are thought not only to be unsuccessful in relieving anxiety and distress, but also maladaptive to psychological functioning (G. E. Valliant, Bond, \& C. O. Valliant, 1986). Hence, as immature defences have been associated with ineffective coping mechanisms, individuals employing immature defences are likely to experience increased levels of anxiety (Bond, 2004).

Recently, research has shown that defence mechanisms may be associated with specific biological responses, while there has been a concurrent interest in the physiological aspects of maternal responsiveness. Mothers with higher baseline HR (heart rate) were found to be more sympathetic to infant cries and better able to discriminate between pain and hunger cries than mothers with lower HR (Stallings, Fleming, Corter, Worthman, \& Steiner, 2001). Studies have effectively used an infant cry to examine maternal physiological responsiveness and attachment (Avery, Lewis, \& Gould, unpublished manuscript), but few studies have considered the role of anxiety and defence mechanisms. Diamond, Hicks, and Otter-Henderson (2006) have shown that avoidantly attached individuals respond to psychological stressors with high physiological responses, as measured by GSR (galvanic skin response) and HR, but low subjective ratings of their reactivity. Such findings suggest that in response to tasks that elicit negative thoughts and feelings, individuals high on avoidant attachment may use the cognitive distortion of defences to avoid the appraisal of negative emotions resulting in a discrepancy between their self-awareness and their physiological response (Jorgensen \& Zachariae, 2006).

Several researchers have studied the biological aspects of parental responsiveness, though the use of an infant cry as the stimulus. One of the key issues in this line of research is how to interpret the changes in physiology. Increases in HR have been interpreted as an aversive or defensive response to the infant cry, whereas HR deceleration has been interpreted as reflecting attention towards the infant (Fox \& Card, 1999). The relationship between HR and parental responsiveness, however, appears quite complex, with other research indicating increased HR is associated with parental experience (Bleichfield \& Moely, 1984). Individuals who were pregnant or had experience with infants were found to demonstrate greater increases in HR and GSR than individuals without children when exposed to the sound of an infant crying (Bleichfield \& Moely, 1984).

Finally, parents reported to have physically abused their children were more likely than control participants or individuals diagnosed with an anxiety disorder to endorse immature defences (Brennan, 
Andrews, Morris-Yates, \& Pollock, 1990). Such findings suggest that these individuals experience high levels of physiological and psychological arousal when exposed to infant/child signals, potentially resulting from ineffective defence mechanisms. Additionally, Crowe, and Zeskind (1992) found that parents who scored low on the CAP (child abuse potential) inventory (Milner \& Wimberley, 1979) had a lower resting HR than those who scored high (indicating a high potential for child abuse). Further, participants who scored high on the CAP responded to cries with a greater increase from their baseline HR and GSR than the low CAP group. This increase may be interpreted as aversive or defensive responding to infant signaling.

No previous studies have examined the interaction of physiological and psychological processes, specifically anxiety and defence style, on parental responsiveness to infant distress. The present study is a preliminary investigation that used an experimental method to investigate the physiological responses of individuals to the sound of both white noise and an infant cry. It was hypothesized that participants would experience the sound of an infant cry as an anxiety provoking stimulus, showing an increase in GSR and HR as compared to the control stimulus. It was also hypothesized that immature and neurotic defence styles would be associated with a greater increase in HR and GSR than mature defence styles in response to an infant cry. As it has been suggested that defence style masks perceived anxiety, it was predicted that the relationship between defence style and HR or GSR in response to an infant cry would be stronger than the relationship between self-reported anxiety and HR or GSR.

\section{Method}

\section{Participants}

Fifty participants were recruited through a convenience sample of university staff and students. Their ages ranged from 19-61 years $(M$ age $=30.29, S D=10.50)$. Of the $38(76 \%)$ female participants, $30(60 \%)$ reported having no children, while eight (16\%) had one or more children. Four males $(8 \%)$ had children, while eight (16\%) did not. All participants were in good general health and did not suffer an intellectual or mental disability. Participants were not paid for involvement in this research. Approval was granted by Deakin University Human Research Ethics Committee.

\section{Materials and Apparatus}

Psychological measures. The STAI (state trait anxiety inventory), a 40-item questionnaire that uses a 4-point Likert scale to assess anxiety, has been widely used in research literature. It is sensitive to state anxiety (transitory episodes of anxiety) and trait anxiety (stable aspects of personality that predispose an individual to experiencing chronic levels of anxiety), providing an indication of how strongly the individual is endorsing state or trait anxiety. This research was primarily interested in using the STAI as a measure of enduring or trait anxiety characteristics. Assessment of trait anxiety possesses adequate test-retest reliability, with coefficients ranging between 0.73 and 0.86 . As expected, test-retest reliability is lower for the changeable construct and state anxiety. Concurrent, content and construct validity support the use of this questionnaire as an adequate means to assess state and trait anxiety levels (Groth-Marnat, 2003). The STAI was scored according to the standard procedure outlined in the manual and standardized scores were obtained using the general norms provided (Spielberger, Goruch, Jacobs, Lusthene, \& Vagg, 1977).

The DSQ-40 (defence style questionnaire-40) is a 40-item questionnaire in which subjects respond to statements representing varying defence mechanisms in a 9-point scale. The DSQ-40 provides empirically 
derived groupings of defence mechanisms that are ranked in an adaptive hierarchy into mature, neurotic and immature defences. It is a continuous measure which provides a value of how strongly the participant endorsed mature, neurotic and immature defences, with a higher score indicating greater endorsement of that defence style. The reliability and validity of the DSQ-40 is supported by its high correlations with the original DSQ, shown to demonstrate sufficient test-retest reliability (Muris \& Merkelbach, 1994). Further, the 40-item version has been shown to discriminate between anxiety patients and controls, bolstering discriminant validity (Bond, 2004). The DSQ-40 was scored following the scoring procedure outlined by Andrews, Singh, and Bond (1993).

Participants also completed a demographic questionnaire requesting information about age, gender and parental status. Its purpose was to assess socio-economic factors, including relationship status, parity and experience caring for an infant, specifically current time spent caring for an infant less than 12 months of age.

Physiological measures. Sympathetic nervous system functioning was measured in the form of GSR (galvanic skin response) and HR derived from the ECG (electrocardiogram). HR is a common measure of autonomic nervous system functioning and well known to increase in response to factors, such as physical exertion, anxiety, arousal, anger or other forms of emotion. GSR has also been shown to be a reliable measure of the autonomic expression of emotion and has been shown to increase with self-reported arousal (Khalfa, Isabelle, Jean-Pierre, \& Mannon, 2002). The ECG and GSR were recorded on LabChart version 6 for Windows, using the Power Lab 26T unit from ADInstruments, with GSR Bio Amplifier. GSR measurement required fitting bipolar finger electrodes to the third and fourth fingers of the participant's non-dominant hand, while disposable adhesive electrodes were used for the ECG recording and were placed in the standard Lead I three-electrode placement (left wrist positive, right wrist negative and the earth electrode connected to the right ankle).

Both ECG and GSR were sampled at a rate of 1,000 samples per second, providing continuous measurement that was then averaged over the experimental phases. HR was calculated from the number of $\mathrm{R}$ waves per minute.

Stimuli. The recording of an infant cry, used for the experimental condition, was obtained from the IADS-2 (2nd edition; international affective digitized sounds-2). The IADS-2 project included a large range of emotionally provoking sounds that were rated in a 9-point scale by 100 college students (with nine representing high arousal or pleasure). The infant cry had a mean rating of 2.75 for pleasure and 6.51 for arousal (Bradley \& Lang, 2007). This recording lasted six seconds and was modified for the current research by extending it to a total duration of one minute. Following the procedure used by Seifritz et al. (2003) and Crowe and Zeskind (1992), each of the five cry segments was followed with a six second interval of silence. The control recording was a neutral recording of one minute of white noise, which is a type of noise frequently used as a control in similar studies (Fleming, Corter, Stallings, \& Steiner, 2002).

\section{Procedure}

A repeated measures design was used, requiring participants be tested on two separate occasions with one session being the presentation of the white noise stimulus and the other testing session involving the presentation of the infant cry stimulus. The second testing session generally took place within one week of the first session, at the same time of day as the first session. With the exception of the stimulus presented, all other aspects of each session were the same.

After signing informed consent, participants were asked to wash their hands to remove perspiration 
build-up that may affect GSR. Collection of the baseline measures then commenced. After cleaning the area with an alcohol swap, a disposable adhesive electrode was placed on both wrists and the right ankle for the measurement of EEG. GSR cuffs were attached to the middle phalange of the middle and ring finger on the participant's non-dominant hand.

Participants were instructed to relax in a comfortable position with their eyes closed and remain as still as possible. Headphones were placed on their ears and continuous recording of GSR and ECG commenced. This recording consisted of three phases: (1) pre-two minutes of baseline data; (2) during — one minute experimental phase recording, while the participants were exposed to either the white noise or the infant cry stimuli; and (3) post — an additional one minute of recovery phase measurement following the end of the stimulus sound.

Participants were given the STAI, DSQ-40, and a demographics questionnaire to complete after the first session and return by the second testing session.

\section{Results}

The HR and GSR data were extracted using the ECG Analysis Module of LabChart 6, and each of these measurements was averaged over the three recording phases and two experimental conditions for each participant. Data was then analyzed by the SPSS (statistical package for the social sciences) statistical program (Version 15.0). Mean and standard deviation values for the three recording phases for GSR and HR were calculated for both stimulus conditions (see Table 1). Descriptive statistics for the three defence styles derived from the DSQ-40 are presented in Table 2, along with trait anxiety as measured by the STAI (with one participant with missing data on this measure).

Table 1

Descriptive Statistics for GSR and HR

\begin{tabular}{|c|c|c|c|c|c|c|c|}
\hline & \multicolumn{4}{|c|}{ Control } & \multicolumn{3}{|c|}{ Cry } \\
\hline & $N$ & Mean & $S D$ & Normality & $N$ & Mean & $S D$ \\
\hline \multicolumn{8}{|c|}{$\overline{\mathrm{GSR}}(\mu \mathrm{sm})$} \\
\hline Pre & 50 & 3.66 & 3.53 & 0.00 & 50 & 3.44 & 2.31 \\
\hline During & 50 & 4.17 & 3.77 & 0.00 & 50 & 4.21 & 2.80 \\
\hline Post & 50 & 4.33 & 3.89 & 0.00 & 50 & 4.29 & 2.91 \\
\hline \multicolumn{8}{|c|}{ HR (BPM) } \\
\hline Pre & 50 & 74.71 & 11.18 & 0.84 & 50 & 73.70 & 11.57 \\
\hline During & 50 & 75.68 & 11.66 & 0.79 & 50 & 72.55 & 11.18 \\
\hline Post & 50 & 75.48 & 10.90 & 0.27 & 50 & 74.64 & 11.57 \\
\hline
\end{tabular}

Table 2

Descriptive Statistics for Defence Style and Trait Anxiety

\begin{tabular}{lccc}
\hline & $N$ & Mean & \\
\hline Defence style & & \\
$\quad$ Mature & 50 & 5.83 & 0.95 \\
Neurotic & 50 & 4.71 & 1.00 \\
Immature & 50 & 3.47 & 0.93 \\
Anxiety & & & \\
Trait & 49 & 38.16 & 7.89 \\
\hline
\end{tabular}

Descriptive statistics presented in Table 2 indicate that as a group, participants in this research endorsed a 
mature defence style more strongly than an immature or neurotic defence style (mature style was the most strongly endorsed of the three for 40 of the 50 participants), which is consistent with the demographic characteristics of the sample.

\section{HR and GSR Reactivity}

Repeated measures ANOVA (analysis of variance) was used to examine change in physiological variables across phase and stimulus conditions. All six GSR variables, with the exception of cry-post, failed to meet the assumption of normality, showing considerable positive skew. A square root transformation was applied to all six GSR variables. The six transformed variables were all normally distributed.

As continuous measurement of GSR often results in "drift" in the data due to the build-up of perspiration on fingers, GSR was initially analyzed with a $2 \times 3$ repeated measures ANOVA in order to include the post-stimulus values in the analysis. This revealed a non-significant main effect for stimulus condition, $F_{(1,49)}=$ $0.03, p>0.05$. The main effect for time of measurement was significant, $F_{(1.56,76.41)}=7.16, p<0.01$, while the interaction was non-significant, $F_{(1.73,84.50)}=0.19, p>0.05$. These results are depicted in Figure 1.

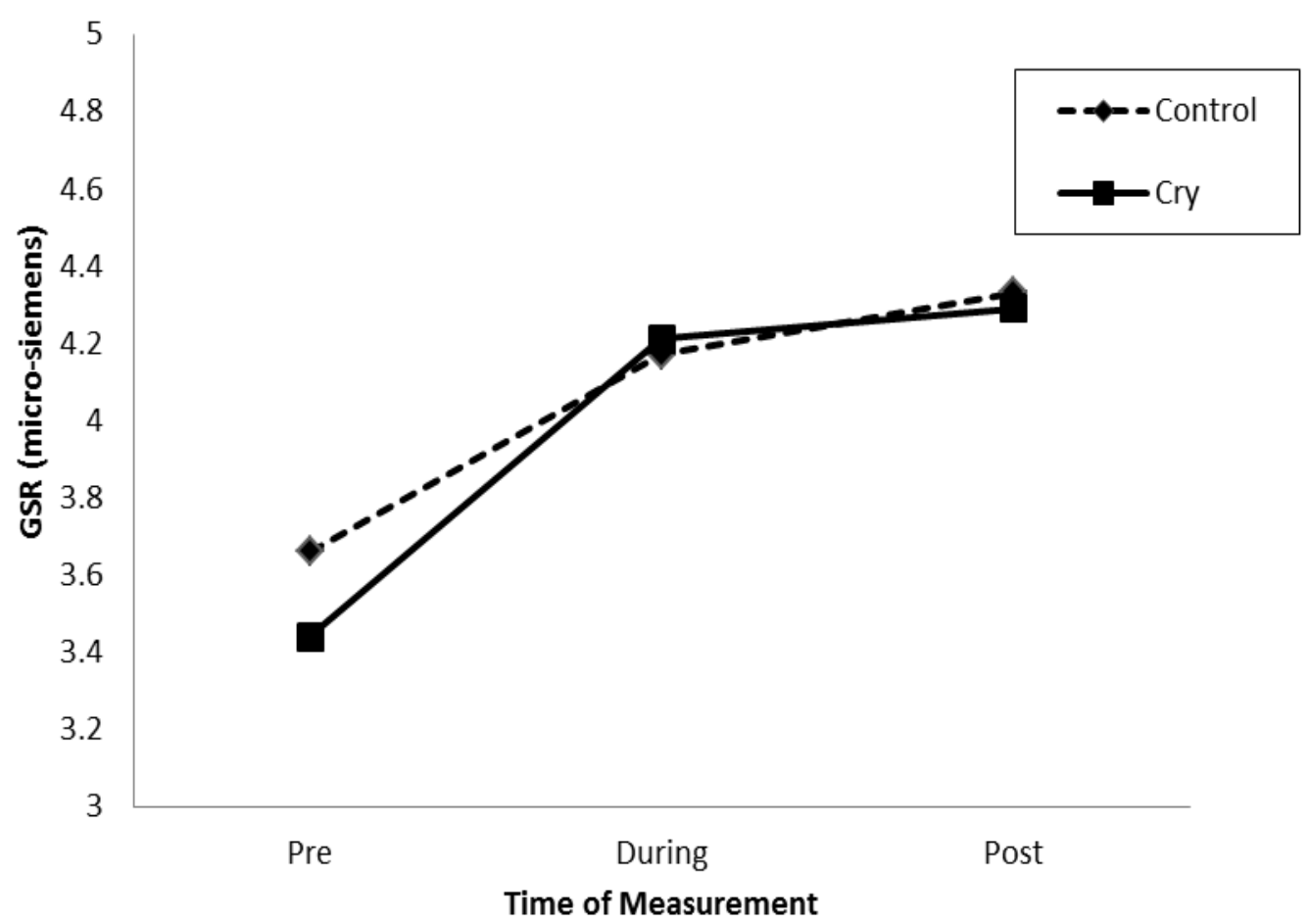

Figure 1. GSR levels across stimulus conditions and experimental phase.

From Figure 1, it can be seen that while there is an increase in mean GSR for both stimulus conditions from the during-stimulus measurement to post-stimulus, this increase is minimal and may be due to random variation or "drift". In contrast, the difference between pre-stimulus (baseline) and during-stimulus measurement for both stimuli is considerably larger. Hence, it was concluded that baseline to stimulus change for both conditions is a genuine change in response to the stimulus and not due simply to a continuous build-up of perspiration on the fingers. The data was re-analyzed with a $2 \times 2$ repeated measures ANOVA to examine only the difference between baseline and stimulus phase for both conditions. 
No significant interaction was found for GSR, $F_{(1,50)}=0.27, p>0.05$. The main effect for condition also failed to meet significance, $F_{(1,50)}=0.06, p>0.05$. A significant main effect was obtained for stimulus phase, $F_{(1,50)}=6.31, p<0.05$. GSR was found to increase in response to both stimulus presentations, but there was minimal difference in the responses across the experimental and control conditions.

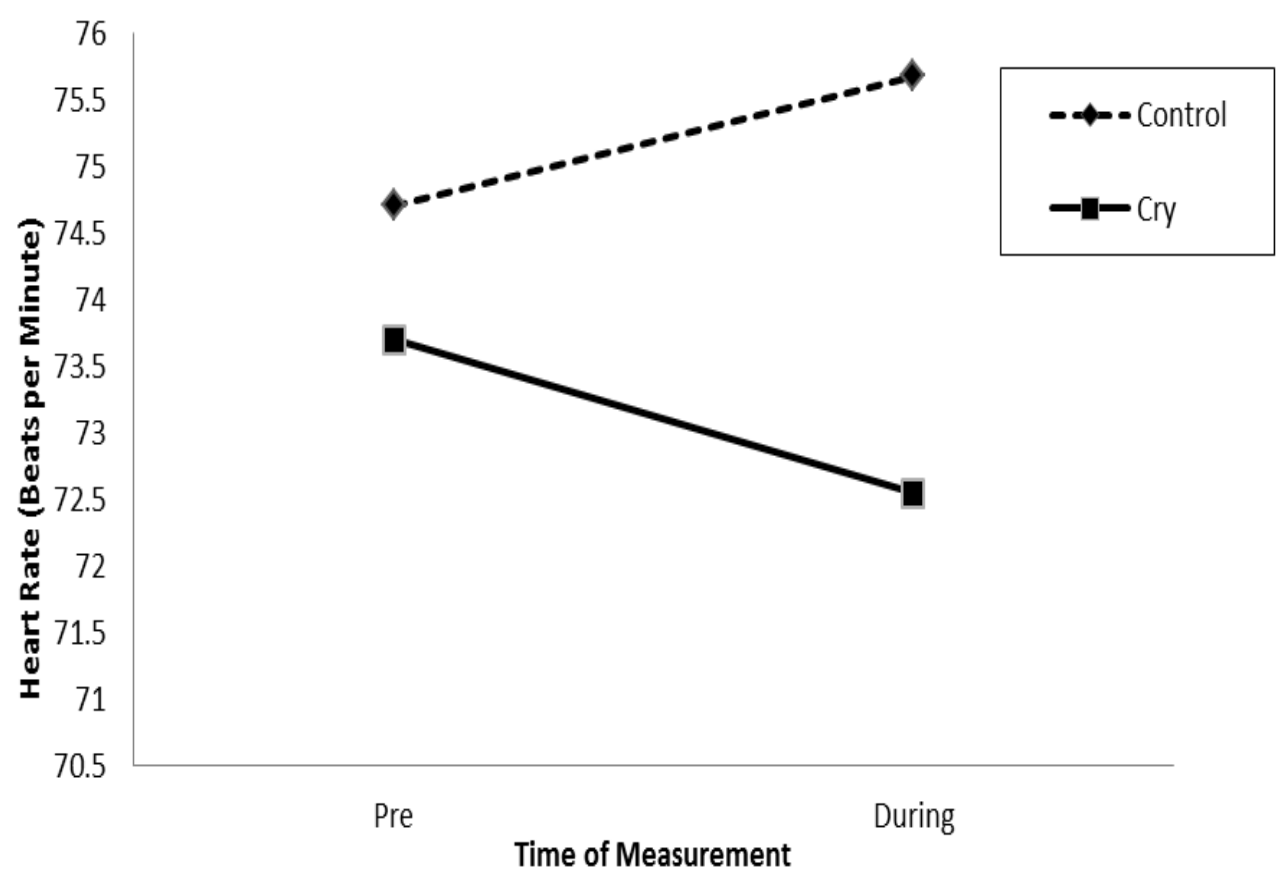

Figure 2. HR levels across stimulus conditions and experimental phase.

A $2 \times 2$ repeated measures, ANOVA, was conducted for HR. All four HR variables of interest (excluding the two post-stimulus measurements) met the assumption for normality. A significant interaction was obtained for HR, $F_{(1,51)}=6.75, p<0.05$. As it can be seen in Figure 2, HR was minimally higher for the control stimulus at baseline $(M=74.71)$ than the cry stimulus $(M=73.70)$. HR then decreased when the cry stimulus was presented $(M=72.55)$ and increased when the control stimulus was presented $(M=75.68)$.

\section{Relationship Between Defence Style, Anxiety and Physiology}

In order to examine the correlations between physiological and psychological measures, single measures for change in both GSR and HR were required. Difference values were calculated for each participant, reflecting the increase in GSR from baseline in response to the cry stimulus, over and above the increase in response to the control stimulus. This was done by subtracting the difference between pre-control and during-control from the difference between pre-cry and during-cry measurement points: change = (during-cry - pre-cry) - (during-control - pre-control). Due to the significant interaction effect noted for HR, this approach was not appropriate for HR. Instead, two change variables were calculated: one reflecting HR acceleration in response to the control stimulus (during-control - pre-control) and one reflecting HR deceleration in response to the cry stimulus (during-cry - pre-control).

A one-tailed Bivariate Pearson Product-Moment Correlation analysis was then conducted to analyze whether differences in endorsement of defence style or anxiety level were significantly correlated with change in GSR or HR (see Table 3). 
Table 3

Correlations Between Psychological Variables and Physiological Response

\begin{tabular}{lcclccc}
\hline \multicolumn{1}{l}{} & 1 & 2 & 3 & 4 & 5 & 6 \\
\hline 1. Mature defence & & & & & & \\
2. Neurotic defence & 0.07 & & & & & \\
3. Immature defence & 0.03 & $0.62^{* *}$ & & & & \\
4. GSR change & 0.24 & $0.38^{* *}$ & $0.47^{* *}$ & & & \\
5. HR control change & -0.16 & 0.05 & 0.07 & 0.15 & & \\
6. HR cry change & -0.11 & -0.11 & 0.15 & 0.08 & 0.09 & $0.26^{*}$ \\
7. Trait anxiety & -0.24 & $0.36^{*}$ & $0.48^{* *}$ & 0.21 & -0.04 & \\
\hline
\end{tabular}

Notes. ${ }^{*}$ Correlation is significant at the level of 0.05 (1-tailed); ${ }^{* *}$ Correlation is significant at the level of 0.01 (1-tailed).

HR acceleration in response to the control stimulus did not correlate with any of the psychological variables. However, HR deceleration in response to the cry sound was significantly positively correlated with trait anxiety, $r_{(49)}=0.26, p=0.037$. HR deceleration was not related to any of the defence styles.

Trait anxiety was significantly correlated with both immature defence style, $r_{(49)}=0.48, p=0.001$, and neurotic defence style, $r_{(49)}=0.36, p=0.006$. Furthermore, the increase in GSR in response to the cry stimulus (over-and-above the response to the control stimulus) was significantly positively correlated with both immature defence style, $r_{(50)}=0.47, p=0.001$, and neurotic defence style, $r_{(50)}=0.38, p=0.003$.

Immature and neurotic defence styles were significantly correlated, $r_{(50)}=0.62, p=0.001$, indicating that the relationship between either independent variables with GSR may be explained by its high correlation with the other independent variables. A hierarchical regression was, therefore, conducted to examine which variable was more predictive of GSR change.

The hierarchical regression was performed for four steps of independent variables as predictors of GSR change. The first block of predictors included age, gender and experience, where experience refers to a categorical variable based on whether or not the participant has children. The second predictor block included trait anxiety. Neurotic defence was entered as the third predictor and finally immature defence was entered into the regression analysis. To screen for univariate outliers, $z$-scores were calculated for all variables. No variable was found to contain $z$-scores higher than 3.29, and therefore, no values were considered outliers (Tabachnick \& Fidell, 1996). Multivariate outliers were screened for using mahalanobis distance. No variable was greater than, or equal to, the critical chi square of 16.81 for six degrees of freedom, at an alpha level of 0.01 (as suggested by Tabachnick \& Fidell, 1996). Assumptions of linearity, normality and homoscedasticity were assessed by examination of scatter-plots of the residuals. These plots displayed a relatively normal pattern, clustering around zero. The ratio of cases to independent variables was not violated. For the analysis to be conducted, five times more cases than predictors must be evident. Seven predictors were included, requiring a minimum of 30 cases (Coakes, Steed, \& Dzidic, 2006). The correlation matrix was examined to check for multi-collinearity. Simple correlations between predictor variables were all low to moderate, reducing the chance of redundancy in the contribution of any one variable to the regression equations. The highest correlation coefficients were between independent variables which had been employed as measures of similar abilities. Age and experience were moderately correlated $(r=0.78)$, as they were immature and neurotic defence $(r=0.64)$. Anxiety correlated with both neurotic $(r=0.36)$ and immature defence $(r=0.48)$. No other correlations between predictor variables 
were obtained.

Table 4

Summary of Hierarchical Multiple Regression Analysis for GSR Change

\begin{tabular}{|c|c|c|c|c|c|}
\hline & $B$ & $S E B$ & $\beta$ & $R^{2}$ & $\Delta R^{2}$ \\
\hline $\begin{array}{l}\text { Step } 1 \\
\end{array}$ & & & & 0.11 & 0.11 \\
\hline Age & -0.07 & 0.05 & -0.35 & & \\
\hline Gender & -0.29 & 0.70 & -0.06 & & \\
\hline Experience & 2.58 & 1.11 & 0.52 & & \\
\hline Step 2 & & & & 0.14 & 0.03 \\
\hline Age & -0.07 & 0.05 & -0.36 & & \\
\hline Gender & -0.29 & 0.70 & -0.06 & & \\
\hline Experience & 2.44 & 1.11 & 0.50 & & \\
\hline Trait anxiety & 0.05 & 0.04 & 0.18 & & \\
\hline Step 3 & & & & 0.23 & $0.09^{*}$ \\
\hline Age & -0.04 & 0.05 & -0.22 & & \\
\hline Gender & -0.41 & 0.67 & -0.08 & & \\
\hline Experience & 2.10 & 1.10 & 0.40 & & \\
\hline Trait anxiety & 0.01 & 0.04 & 0.04 & & \\
\hline Neurotic & 0.73 & 0.32 & 0.34 & & \\
\hline Step 4 & & & & 0.31 & $0.07^{*}$ \\
\hline Experience & -0.02 & 0.05 & -0.10 & & \\
\hline Gender & -0.53 & 0.65 & -0.11 & & \\
\hline Age & 1.71 & 1.05 & 0.35 & & \\
\hline Trait anxiety & -0.02 & 0.04 & -0.09 & & \\
\hline Neurotic & 0.34 & 0.36 & 0.16 & & \\
\hline Immature & 0.92 & 0.43 & 0.39 & & \\
\hline
\end{tabular}

Note. ${ }^{*} p<0.05$.

As shown in Table 4, the demographic variables were not significantly associated with GSR change. Age, gender and experience failed to reach significance $\left(R^{2}=0.11, F_{(3,45)}=1.88, p>0.05\right)$, as did trait anxiety $\left(R^{2}=0.14, F_{(1,44)}=1.50, p>0.05\right)$. While step 3 revealed that neurotic defence was a significant predictor of GSR $\left(R^{2}=0.23, F_{(1,43)}=5.11, p>0.05\right)$, step 4 revealed that immature defence was also a significant predictor of GSR $\left(R^{2}=0.31, F_{(1,42)}=4.45, p<0.05\right)$. The inclusion of immature defence, however, rendered neurotic defence a non-significant predictor of change in GSR levels $(p<0.05)$. Hence, immature defence was found to account for $31 \%$ of the variance in GSR increase unique to the cry stimulus, partially supporting hypothesis 2 that immature and neurotic defence would be associated with increased physiological responsiveness.

\section{Discussion and Conclusions}

The current study was primarily interested in the relationship between physiological responses to the sound of an infant cry and the psychological variables of defence style and anxiety. It was hypothesized that participants would demonstrate a physiological response to the stimulus condition. Results partially support this prediction. While GSR was found to increase in response to the sound of an infant crying, a similar increase in GSR was noted in response to the control stimulus, indicating that participants found both stimuli anxiety provoking. In contrast, the HR response to the two stimuli was significantly different. While it was 
hypothesized that HR would increase in response to the infant cry, HR increased in response to the white noise but decreased in response to the infant cry.

The hypothesis that immature and neurotic defence would be associated with greater increases in physiological responsiveness was supported. A significant and positive correlation was found for both immature and neurotic defence with GSR responsiveness, indicating that individuals who score high on neurotic or immature defence styles experience the sound of an infant crying as anxiety provoking and possibly aversive. Further, there was no correlation between mature defence and change in physiological arousal, indicating that participants endorsing mature defence styles do not demonstrate a systematic increase in GSR when listening to the sound of an infant crying, over and above that of listening to white noise. Such individuals mobilize more adaptive responses to the stress inherent in hearing an infant cry and could be considered to successfully alleviate their anxiety and, therefore, do not experience the sound as aversive or excessively distressing. Immature defensiveness was also shown to be a unique and quite strong predictor of change in GSR levels, accounting for a substantial proportion of the variance in GSR responses.

Interestingly, no relationship was found between immature or neurotic defence with change in HR. Such findings indicate that endorsement of less mature defences was not related to HR change in the present study. HR deceleration in response to the cry stimulus did show a mild positive correlation with trait anxiety. There may be a number of explanations for this finding. Firstly, previous research has demonstrated that experienced parents will show increases in HR in response to an infant cry, while inexperienced parents will show decreases in HR (Bleichfield \& Moely, 1984). As many participants in the current study did not have parental experience, the current findings may be unduly influenced by the lack of parental experience in our sample. Future research should examine this in more detail, as it is possible that a correlation between defence style and HR may emerge when controlling for parental experience. Furthermore, inadequate power may have contributed to the lack of significant correlations between immature and neurotic defence, as the majority of participants were also classified as employing mature defence styles.

It was also hypothesized that defence style would show a stronger relationship to HR and GSR than anxiety. This prediction was partially supported. No significant correlation was found between trait anxiety and change in GSR. Furthermore, only a mild correlation was found between trait anxiety and HR deceleration. Defence style, however, did correlate significantly with change in GSR, indicating that immature and neurotic defence were ineffective in alleviating physiological anxiety in response to the infant cry, while perceived anxiety was not related to physiological arousal.

The current study is one of the first to demonstrate that variation in physiological responsiveness is largely related to the effectiveness of defence mechanisms employed. Findings obtained for GSR are supportive of a theory proposed by Diamond et al. (2006) who suggested that the use of ineffective defence mechanisms, in response to tasks that elicit negative thoughts and feelings, is correlated with increased GSR. While the current study found GSR to increase in response to both the cry and control stimuli, the increase in GSR unique to the infant cry was moderately correlated with immature defensiveness. Furthermore, this relationship between defence style and GSR increase was evident even after controlling for age, anxiety and experience with infants.

Interestingly, the current study found HR to decrease in response to the infant cry, reflecting a significant finding in the opposite direction to that predicted. While this change was mildly related to trait anxiety, it was not found to be associated with defence style, suggesting that decelerated HR in response to the sound of an infant cry is not affected by defence style. Fox and Card (1999) suggested that HR deceleration indicates 
attention toward the infant's signals, while HR acceleration indicates an aversive and defensive reaction to the cry. In addition, other studies have found increases in HR in response to an infant cry for individuals who have had experience with children or are pregnant (Bleichfield \& Moely, 1984). As few participants in our study were parents, future research may endeavour to examine whether parents are less likely to endorse defence mechanisms, when exposed to an infant cry, than those who are not parents. In contrast, other literature has suggested that HR deceleration is produced in anticipation of, and during the processing of, emotional stimuli (Poli, Sarlo, Bortoletto, Buodo, \& Palomba, 2007). Hence, the finding that HR decreased in response to the infant cry may be a consequence of the fact that the participants were anticipating and processing the infant cry. Results in the current study may also have been influenced by the relatively small number of participants who scored higher on immature compared to mature defence styles. This may be addressed in future research by testing individuals from a broader sample.

This preliminary study demonstrates that an interaction between biology and psychology is responsible for some of the variations in how individuals respond to environmental stimuli. The findings obtained in this study indicate that an individual's defence style can predict a substantial proportion of the variance in their GSR response when exposed to the sound of an infant cry. It must be noted, however, that a number of participants commented the cry sounded like that of an older child rather than an infant and that the child did not sound particularly distressed. A stronger cry stimulus may have the potential to elicit a significant interaction between the two stimulus conditions, leading to a stronger relationship between the change score and defence style. In addition, future research may attempt to control for systematic differences across testing sessions by employing different control stimuli and counter balancing the order of stimulus presentation. However, the fact that counterbalancing was not utilized in the present study supports the strength of the findings obtained. As the first testing session involved stress associated with novelty, the physiological measures were expected to be inflated for this session, artificially inflating stress responses to the control stimulus. The fact that significant differences were obtained, despite this artificially inflated physiological response to the control conditions is a testament to the significance of these results. As this is one of the first experiments to explicitly examine the relationship between defence style and physiological responsiveness, further research is warranted. Not only may this provide insight into variation in physiological and psychological variables amongst the population, but such research may add to the understanding of particularly unusual parental responses to infant signals.

\section{References}

Andrews, G., Pollock, C., \& Stewart, G. (1989). The determination of defence style by questionnaire. Archives of General Psychiatry, 46, 455-460.

Andrews, G., Singh, M., \& Bond, M. (1993). The defence style questionnaire. Journal of Nervous and Mental Disease, 181(4), 246-256.

Avery, M., Lewis, A. J., \& Gould, E. L. (unpublished manuscript). Adult attachment style and physiological reactivity to infant distress.

Barry, P. D., \& Farmer, S. (2001). Mental health and mental illness. Philadelphia: Lippinncott Williams and Wilkins.

Bartholomew, K., \& Horowitz, L. (1991). Attachment styles among young adults: A test of a four-category model. Journal of Personality and Social Psychology, 61, 226-244.

Bleichfeld, B., \& Moely, B. E. (1984). Psychophysiological responses to an infant cry: Comparison of groups of women in different phases of the maternal cycle. Developmental Psychology, 20, 1082-1091.

Bond, M. (2004). Empirical studies of defence style: Relationships with psychopathology and change. Harvard Review of Psychiatry, 12, 263-278. 
Brennan, J., Andrews, G., Morris-Yates, A., \& Pollock, C. (1990). An examination of defence styles in parents who abuse children. The Journal of Nervous and Mental Disease, 178, 592-595.

Bradley, M. M., \& Lang, P. J. (2007). The international affective digitized sounds (2nd ed., IADS-2): Affective ratings of sounds and instruction manual. Technical Report B-3. F. L.: University of Florida, Gainesville.

Brown, L. L., Tomarken, A. J., Orth, D. N., Loosen, P. T., Kalin, N. H., \& Davidson, R. J. (1996). Individual differences in repressive-defensiveness predict basal salivary cortisol levels. Journal of Personality and Social Psychology, 70, 362-371.

Coakes, S., Steed, L., \& Dzidic, P. (2006). SPSS version 13.0 for windows: Analysis without anguish. Wiley-India.

Cohen, B. J. (2003). Theory and practice of psychiatry. London: Oxford University Press.

Crowe, H. P., \& Zeskind, P. S. (1992). Psychophysiological and perceptual responses to infant cries varying in pitch: Comparison of adults with low and high scores on the child abuse potential inventory. Child Abuse and Neglect, 16, 19-29.

Diamond, L. M., Hicks, A. M., \& Otter-Henderson, K. (2006). Physiological evidence for repressive coping among avoidantly attached adults. Journal of Social and Personal Relationships, 23, 205-229.

Donavan, W. L., \& Leavitt, L. A. (1989). Maternal self-efficacy and infant attachment: Integrating physiology, perceptions and behaviour. Child Development, 60, 460-472.

Fleming, A. S., Corter, C., Stallings, J., \& Steiner, M. (2002). Testosterone and prolactin are associated with emotional responses to infant cries in new fathers. Hormones and Behavior, 42, 339-413.

Fox, N. A., \& Card, J. A. (1999). Psychophysiology \& attachment. In J. Cassidy, \& P. R. Shaver (Eds.), Handbook of attachment, theory, research and clinical applications (pp. 226-240). New York: Guilford Press.

Groth-Marnat, G. (2003). Brief instruments for treatment planning, monitoring and outcome assessment: Handbook of psychological assessememt (4th ed.). New York: John Wiley \& Sons.

Huprich, S. K. (2008). Psychodynamic therapy: Conceptual and empirical foundations. Kentucky, USA: Routledge.

Jorgensen, M. M., \& Zachariae, R. (2006). Repressive coping style and autonomic reactions to two experimental stressors in men and women. Scandinavian Journal of Psychology, 47, 137-148.

Khalfa, S., Isabelle, P., Jean-Pierre, B., \& Mannon, R. (2002). Event related skin conductance responses to musical emotions in humans. Neuroscience Letter, 328, 145-149.

Lewis, A., \& White, J. (2009). The defense mechanisms of homophobic adolescent males: A descriptive discriminant analysis. Journal of Adolescence, 32, 435-441.

McGregor, P. K., \& Peake, T. M. (2000). Communication networks: Social environments for sending and receiving signaling behavior. Acta Ethol, 2, 71-81.

Mikulincer, M., \& Orbach, I. (1995). Attachment styles and repressive defences: The accessibility and architecture of affective memories. Journal of Personality and Social Psychology, 68, 917-925.

Milner, J. S., \& Wimberley, R. C. (1979). An inventory for the identification of child abusers. Journal of Clinical Psychology, 35, 95-100.

Muris, P., \& Merckelbach, H. (1994). Defence style, trait anxiety, worry and bodily symptoms. Personality and Individual Differences, 15, 349-341.

Nishimura, R. (1998). Study of the measurement of defence style using bond's defence style questionnaire. Psychiatry and Clinical Neurosciences, 52, 419-424.

Polan, H. J., \& Hofer, M. A. (1999). Psychobiological origins of infant attachment and separation responses. In J. Cassidy, \& P. R. Shaver (Eds.), Handbook of attachment, theory, research and clinical applications (pp. 163-244). New York: Guilford Press.

Poli, S., Sarlo, M., Bortoletto, M., Buodo, G., \& Palomba, D. (2007). Stimulus-preceding negativity and heart rate changes in anticipation of affective pictures. International Journal of Psychophysiology, 65(1), 32-39.

Seifritz, E., Esposito, F., Neuhoff, J. G., Luthi, A. ... Di Salle, F. (2003). Differential sex independent amygdala response to infant crying and laughing in parents versus nonparents. Biological Psychiatry, 54(12), 1367-1375.

Spielberger, C. G., Goruch, R. L., Jacobs, G. A., Lusthene, R. E., \& Vagg, P. R. (1977). Test manual for the state-trait anxiety inventory. Palo Alto, California: Consulting Psychologists Press.

Stallings, J., Fleming, A. S., Corter, C., Worthman, C., \& Steiner, M. (2001). The effects of infant cries and odour on sympathy, cortisol, and autonomic responses in new mothers and non postpartum women. Parenting: Science and Practice, 1, 71-100.

Tabachnick, B. G., \& Fidell, L. S. (1996). Using multivariate statistics (3rd ed.). New York: Harper Collins.

Valliant, G. E., Bond, M., \& Valliant, C. O. (1986). An empirically validated hierarchy of defence mechanisms. Archives of General Psychiatry, 43, 786-794. 